\title{
The Changing Periọd of R Hya
}

\author{
Albert A. Zijlstra \\ UMIST, Department of Physics, P.O. Box 88, Manchester, UK \\ Timothy R. Bedding \\ University of Sydney, School of Physics, Sydney, NSW 2006, Australia
}

\begin{abstract}
We discuss the period evolution of the Mira variable $\mathrm{R}$ Hya. We show, using observations dating back to 1662, that it has evolved from a stable 500-day period to a stable 380-day period. The onset of the period evolution coincided with a sharp decline in mass-loss rate. We suggest that mass-loss fluctuations over a period of a few hundred years may be common in Miras, and relate to a non-linear pulsation instability.
\end{abstract}

\section{R Hya: a Mira with declining period}

$\mathrm{R}$ Hya is an unusual Mira variable, whose period has been declining steadily for over a century. Wood \& Zarro (1981) model the period variation assuming a recent (AD1700) thermal pulse. $\mathrm{R}$ Hya is the third oldest variable star known, discovered in 1662. It was observed intermittently until 1850. After 1850, more extensive observations were made. We have analyzed all available historical data, and were able to deduce periods going back to the earliest observations.

Fig. 1 shows the period evolution. The period was stable after 1662 , at 495 days, and begin to decrease around 1770. The decrease continued until 1950 after which the period stabilized at 385 days. The period jitter since 1950 is within the normal range shown by Mira variables.

Two models can explain the behaviour of $\mathrm{R}$ Hya. The first is a recent thermal pulse. However, this does not match the stable periods before and after the decline. The other model concerns envelope relaxation, caused by a change in amplitude of the non-linear Mira pulsation (Ya'ari \& Tuchman 1996). Icke at al. (1992) show that non-linear (chaotic) behaviour increases with decreasing envelope mass, i.e. near the tip of the AGB.

\section{Mass loss and the period variation}

The IRAS LRS spectrum of R Hya shows a strong dust continuum, but without a silicate feature. The silicate emission forms close to the star and its absence indicates a declining mass loss. Hashimoto et al. (1998) fit the spectrum with a detached shell with an inner radius $R_{i}=6 \times 10^{15} \mathrm{~cm}$. Using the CO expansion velocity of $7.5 \mathrm{~km} / \mathrm{s}$, the mass-loss rate decreased sharply around AD 1750, by at least a factor of 10. The relation between period and mass-loss rate of Vassiliadis 


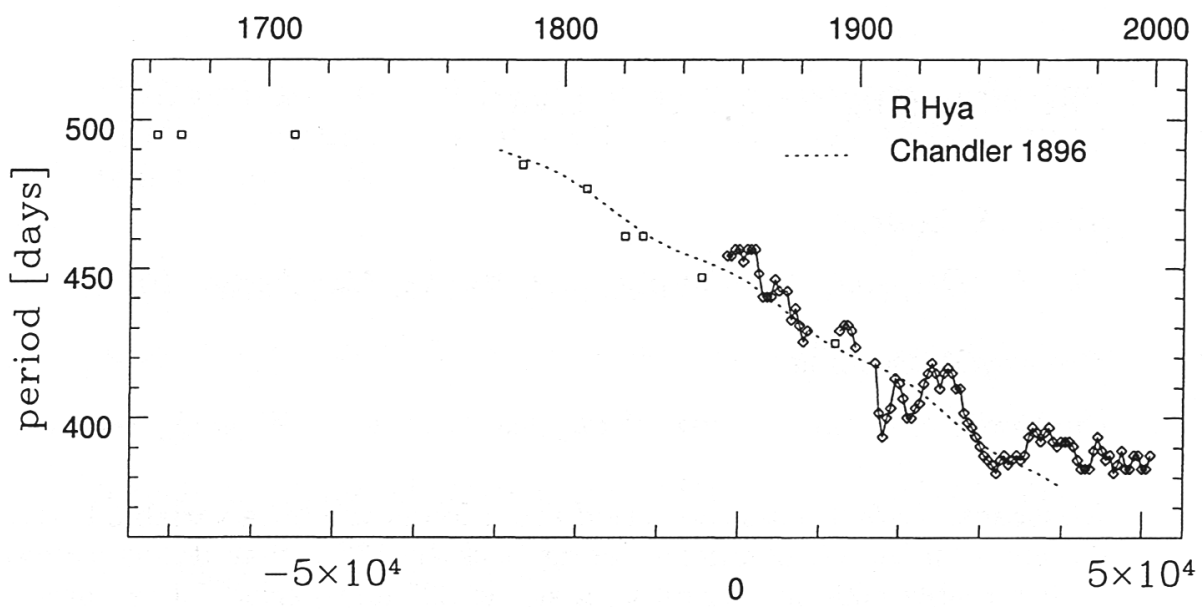

days [JD-240000]

Figure 1. Period evolution between 1662 and 2000. The dashed line is the fit proposed by Chandler in 1896.

\& Wood (1993) predicts a 20 -fold reduction in $\dot{M}$ for R Hya. The mass-loss relation of Blöcker (1995) predicts only a minor reduction of the mass-loss rate of R Hya during its period decline. The observed mass-loss decline supports the formalism of Vassiliadis \& Wood. A more extended detached IRAS shell shows that $\mathrm{R}$ Hya has experienced another mass loss reduction $\sim 3000 \mathrm{yr}$ ago.

Several post-AGB stars show distinct rings around them, indicative of timevariable mass loss at a time scale of $\sim 500 \mathrm{yr}$. $R$ Hya is the only star known showing mass loss changes on this time scale. If due to envelope relaxation, its behaviour is likely periodic, and this can become more pronounced while the envelope mass is further reduced (Icke et al. 1992). R Hya-type behaviour is a possible candidate for the origins of the circumstellar post-AGB rings.

\section{References}

Blöcker T., 1995, A\&A, 297, 727

Chandler, S.C. 1896, AJ, 16, 145

Hashimoto O., Izumiura H., Kester D. J. M., Bontekoe T. R., 1998, A\&A, 329, 213

Icke V., Frank A., Heske A., 1992, A\&A, 258, 341

Vassiliadis E., \& Wood P. R., 1993, ApJ, 413, 641

Wood P. R., \& Zarro D. M., 1981, ApJ, 247, 247

Ya'Ari A., \& Tuchman Y., 1996, ApJ, 456, 350 